\title{
Circulating Interferon- $\lambda 3$, Responsiveness to HBV Vaccination, and HBV/HCV Infections in Haemodialysis Patients
}

\author{
Alicja E. Grzegorzewska, ${ }^{1}$ Monika K. Świderska, ${ }^{2}$ \\ Adrianna Mostowska, ${ }^{3}$ and Pawel P. Jagodziński ${ }^{3}$ \\ ${ }^{1}$ Chair and Department of Nephrology, Transplantology and Internal Diseases, Poznan University of Medical Sciences, Poznan, Poland \\ ${ }^{2}$ Student Nephrology Research Group, Chair and Department of Nephrology, Transplantology and Internal Diseases, \\ Poznan University of Medical Sciences, Poznan, Poland \\ ${ }^{3}$ Department of Biochemistry and Molecular Biology, Poznan University of Medical Sciences, Poznan, Poland \\ Correspondence should be addressed to Alicja E. Grzegorzewska; alicja_grzegorzewska@yahoo.com
}

Received 29 May 2017; Revised 16 August 2017; Accepted 30 August 2017; Published 31 October 2017

Academic Editor: Naohiko Masaki

Copyright (C) 2017 Alicja E. Grzegorzewska et al. This is an open access article distributed under the Creative Commons Attribution License, which permits unrestricted use, distribution, and reproduction in any medium, provided the original work is properly cited.

\begin{abstract}
The IFN- $\lambda 3$ gene (IFNL3) plays a role in HCV clearance. We investigated circulating IFN- $\lambda 3$ and IFNL3 SNPs in haemodialysis patients who differed in their response to HBV vaccination and their HBV/HCV infection status. In 201 patients, plasma IFN- $\lambda 3$ was determined using ELISA. IFNL3 SNPs (rs12979860, rs8099917) were genotyped using HRM analysis. Differences in IFN- $\lambda 3$ levels were shown between responders and nonresponders to HBV vaccination and between HBsAg-positive patients and those who developed anti-HBs after infection and became HBsAg negative. HBV vaccine responders without HCV resolution revealed lower IFN- $\lambda 3$ than noninfected responders. HBsAg/HCV RNA-positive subjects showed lower IFN- $\lambda 3$ than patients positive only for HCV RNA or subjects who resolved both infections. Circulating IFN- $\lambda 3$ correlated positively with anti-HBs and negatively with positive HCV RNA testing in the adjusted regression analyses. HBV vaccine nonresponders, HBsAg-positive patients, and subjects with replicating HCV composed a group with unfavourable outcomes. Responders to HBV vaccination, subjects who became HBsAg negative, and those who cleared HCV were analysed as having favourable outcomes. The latter showed higher IFN- $\lambda 3$ but did not differ in distribution of IFNL3 SNPs compared with subjects with unfavourable outcomes. Higher IFN- $\lambda 3$ concentrations are associated with response to HBV vaccination, self-limited HBV infection, and HCV resolution.
\end{abstract}

\section{Introduction}

The prevalence rates of hepatitis B virus (HBV) and hepatitis $\mathrm{C}$ virus (HCV) infections differ worldwide. Subjects positive for $\mathrm{HBV}$ surface antigen ( $\mathrm{HBsAg}$ ) were found to compose approximately $0.20 \%$ of the Mexican population and $22.38 \%$ of South African inhabitants [1]. Approximately 3\% of the world population is infected with HCV [2]. The prevalence of infection is higher in patients with altered immunocompetence. Among immunocompromised individuals, there are patients with severe renal functional damage that requires regular dialysis treatment. In Poland, antibodies against $\mathrm{HCV}$ (anti-HCV) were shown in $7.9 \%$ and $\mathrm{HBsAg}$ positivity in $3.0 \%$ of haemodialysis (HD) recipients [3], whereas in the general population the respective percentages were $1 \%$ [4] and $0.42 \%$ [1]. Although $\mathrm{HBV}$ vaccination is now a common practice, dialysis patients are less likely to develop antibodies to HBsAg (anti-HBs) after immunization due to impaired immune system function and tend to lose them faster than healthy vaccinated subjects $[5,6]$. A uremic milieu with a related alteration of immunocompetence also promotes the maintenance of $\mathrm{HBV} / \mathrm{HCV}$ replication $[7,8]$.

Interferon- (IFN-) $\lambda$ s are potent antiviral cytokines. IFN$\lambda 3$, also referred to as interleukin- (IL-) 28B, was discovered in early 2003 by two independent groups $[9,10]$. It comprises type III subset of IFNs. IFN- $\lambda$ s, which are directly produced in response to HCV infection, inhibit HCV replication [1113] and are associated with spontaneous resolution and 
successful treatment of HCV infection [14]. IFN- $\lambda$ s also inhibit HBV replication in a differentiated murine hepatocyte cell line [12]. In a nonuremic Han Chinese population, serum IFN- $\lambda 3$ levels were lower in patients with chronic HBV infection than in subjects with self-limited HBV infection or in healthy subjects [15]. Our earlier study on HD patients demonstrated that circulating IFN- $\lambda 3$ correlates with antiHBs production after $\mathrm{HBV}$ vaccination and infection, but HCV-infected subjects were not excluded from the examined groups and were not separately analysed [16].

IFN- $\lambda 3$ is a protein product of the IFN- $\lambda 3$ gene (IFNL3) clustered on chromosome 19 (19q13.13 region, contig AC011445.6). Major homozygosity in IFNL3 rs12979860 is associated with spontaneous HCV clearance $[17,18]$ and resolution of $\mathrm{HCV}$ infection following treatment with pegylated IFN- $\alpha$ and ribavirin $[18,19]$. A meta-analysis by Tang et al. [20] found no association between IFNL3 rs12979860 C/T and persistent $\mathrm{HBV}$ infection risk.

Our aim was to investigate circulating IFN- $\lambda 3$ and single nucleotide polymorphisms (SNPs) of IFNL3 in HD patients who differed in their response to $\mathrm{HBV}$ vaccination and occurrence of $\mathrm{HBV} / \mathrm{HCV}$ infections.

\section{Patients and Methods}

2.1. Patients and Controls. A group of $201 \mathrm{HD}$ patients was included in a cross-sectional study. A majority of the patients were dialysed in two dialysis facilities; however, due to the small number of subjects infected with HBV and/or $\mathrm{HCV}$ at these two centres, enrolment of patients showing nonresponsiveness to $\mathrm{HBV}$ vaccination (anti-HBs $<10 \mathrm{IU} / \mathrm{L}$ ), as well as patients with positive $\mathrm{HBV} / \mathrm{HCV}$ seromarkers, was also performed at other 18 dialysis centres in the Greater Poland region of Poland. The inclusion criteria were as follows:

(1) age over 18 years,

(2) established HBV/HCV seromarkers,

(3) established status as a responder or nonresponder to $\mathrm{HBV}$ vaccination according to the Advisory Committee on Immunization Practices of the US Centers for Disease Control and Prevention [21],

(4) stable clinical condition for at least 2 months prior to enrolment.

Corticosteroid therapy and cachectic conditions causing decreases in serum proteins (neoplasms, enteropathies, and liver cirrhosis), as well as antiviral treatment prior to or at the time of enrolment, were exclusion criteria.

All patients were treated with intermittent HD three times a week, with dialysis sessions lasting approximately four hours each, using low-flux $\mathrm{HD}$, high-flux $\mathrm{HD}$, or on-line haemodiafiltration (HDF).

$\mathrm{HD}$ patients who were responders to $\mathrm{HBV}$ vaccination were tested for anti-HBs titres every 6 months. If their antiHBs titres decreased below $10 \mathrm{IU} / \mathrm{L}$ or were approaching $10 \mathrm{IU} / \mathrm{L}$, booster vaccine doses were administered to keep the anti-HBs titres over $10 \mathrm{IU} / \mathrm{L}$, the level that is considered protective against $\mathrm{HBV}$ infection [21].
HD patients who did not respond to HBV vaccination, those who remained $\mathrm{HBsAg}$ positive after infection, and those with replicating $\mathrm{HCV}$ composed a group with unfavourable outcomes with respect to $\mathrm{HBV}$ vaccination and $\mathrm{HBV} / \mathrm{HCV}$ infections ( $n=63,31.3 \%$ of the total). HD subjects who were responders to $\mathrm{HBV}$ vaccination, those who became HBsAg negative after HBV infection, and those who spontaneously cleared HCV were included in a group with favourable outcomes ( $n=138,68.7 \%$ of the total).

Healthy subjects recruited among medical workers and their friends $(n=28)$ having anti-HBs $\geq 10 \mathrm{IU} / \mathrm{L}$ after HBV vaccination served as controls.

2.2. Laboratory Methods. The blood samples for laboratory measurements were collected before the midweek dialysis session during routinely performed periodical blood examinations appropriate for HD patients.

Plasma IFN- $\lambda 3$ concentration was determined with an enzyme-linked immunosorbent assay (ELISA) kit specific for IFN- $\lambda 3$ (Human Interleukin 28B ELISA Kit, Sunred Biological Technology Co., Ltd., Shanghai, China). The ELISA was performed according to the manufacturer's instructions, and the samples were measured in an ELISA plate reader (Infinite F50, Tecan Group Ltd., Männedorf, Switzerland). Absorbance readings were taken at $450 \mathrm{~nm}$, and results for the test samples were extrapolated from standard curves. The sensitivity of the IFN- $\lambda 3$ ELISA was $0.65 \mathrm{ng} / \mathrm{L}$. The intra-assay coefficient of variation (CV) was $<10 \%$, and the interassay CV was $<12 \%$.

Anti-HBs titres were determined with microparticle enzyme immunoassay (MEIA) technology (ABBOTT, Germany) or the chemiluminescent microparticle immunoassay (CMIA) method (ABBOTT, Ireland). Titres equal to or exceeding 1,000 IU/L were reported as 1,000 IU/l.

Other laboratory parameters were determined using routine methods.

2.3. Genotyping. The IFNL3 SNPs ( $\mathrm{rs} 12979860 \mathrm{C}>\mathrm{T}$ and rs8099917 T>G) of all HD patients were genotyped using a high-resolution melting (HRM) curve analysis. The analysis was performed on a LightCycler 480 system (Roche Diagnostics, Mannheim, Germany) with 5x HOT FIREPol EvaGreen HRM Mix (Solis BioDyne, Tartu, Estonia) as previously described [16]. For quality control, approximately $10 \%$ of the randomly chosen samples were regenotyped using the same genotyping method; the concordance rate was $100 \%$. Genotyping failed for one sample, and that sample was excluded from further statistical analyses.

2.4. Statistical Methods. The results are presented as percentage for categorical variables and medians and range (minimum-maximum) as continuous variables were nonnormally distributed as determined by the Shapiro-Wilk test.

The Mann-Whitney $U$ test was used to compare continuous variables. The power of tests comparing IFN- $\lambda 3$ concentrations or anti-HBs titres in subgroups is shown. Adjustment for possible confounding variables was performed using logistic regression. In statistical comparisons, anti-HBs titres $\geq 1,000 \mathrm{IU} / \mathrm{L}$ were taken as equal to $1,000 \mathrm{IU} / \mathrm{L}$. Spearman's 
rank-order correlation coefficient was used to show correlations between selected variables. Pearson's chi-squared test or Fisher's exact test was applied for the comparison of dichotomous variables. The Cochran-Armitage trend test was used to show the significance of trends in the distribution of genotype frequencies between studied groups.

Stepwise logistic regression with backward elimination was applied to select significant variables among other possible determinants of a tested phenotype. A receiver operating characteristic (ROC) curve was plotted to show the area under the curve (AUC) as a measure of the accuracy of the model.

Linear regression was used to determine the associations among circulating IFN- $\lambda 3$ concentrations and patient characteristics. Stepwise linear regression with backward elimination was applied to select independent correlates of circulating IFN- $\lambda 3$.

A $P$ value less than 0.05 was considered significant, but the Bonferroni correction was applied for evaluation of IFNL3 associations. All probabilities were two-tailed.

The previously mentioned statistical analyses were performed using Statistica version 12 (Stat Soft, Inc., Tulsa, OK, USA), R software version 3.4.0 [22], and $\mathrm{G} *$ Power 3.1.9.2 (Franz Faul, Universitat Kiel, Germany).

Haplotype frequencies were estimated using the software Haploview 4.2 (http://www.broad.mit.edu/mpg/haploview/). Statistical significance was assessed using the 1000-fold permutation test.

2.5. Ethical Approval of Research. The study design was approved by the Institutional Review Board of Poznan University of Medical Sciences, Poland. Written informed consent was obtained from all study participants (201 HD patients and 28 controls).

\section{Results}

3.1. HBV and HCV Status of HD Patients. Among the HD patients, 102 (50.7\%) were uninfected by hepatotropic viruses, 32 (15.9\%) had been exposed to HBV, 35 (17.4\%) had been infected with $\mathrm{HCV}$, and 32 (15.9\%) showed seromarkers of both infections (Table 1). HBsAg positivity was shown in 8 (4.0\%) patients, and anti-HCV positivity was demonstrated by 67 (33.3\%) subjects; among them, 39 (57.4\%) had replicating $\mathrm{HCV}$ (median viral load $2.43 E 5 \mathrm{IU} / \mathrm{mL}$, the range of $0.38 E 5 \mathrm{IU} / \mathrm{mL}-7.71 E 5 \mathrm{IU} / \mathrm{mL})$ and $28(41.8 \%)$ spontaneously resolved their $\mathrm{HCV}$ infections (viral load undetectable).

3.2. IFN- $\lambda 3$ and Anti-HBs in HD Patients and Healthy Subjects. Circulating IFN- $\lambda 3$ concentrations, anti-HBs titres, prevalence of nonresponders to $\mathrm{HBV}$ vaccination, and prevalence of subjects with anti-HBs titre $\geq 1000 \mathrm{IU} / \mathrm{L}$ among subgroups of $\mathrm{HD}$ patients stratified by $\mathrm{HBV} / \mathrm{HCV}$ seromarkers and in healthy subjects are shown in Table 1. Statistical comparisons of variables shown in Table 1 are presented in Table 2.

HBV vaccine responders among noninfected HD patients showed higher IFN- $\lambda 3$ than healthy responders. Anti-HBs titres in these groups were not significantly different (group 2 versus group 22 in Tables 1 and 2).

In the HD group, significant differences in circulating IFN- $\lambda 3$ were shown between noninfected responders and nonresponders to $\mathrm{HBV}$ vaccination (group 2 versus group 3 in Tables 1 and 2) as well as between HBsAg-positive patients and those who developed anti-HBs after HBV infection and became HBsAg negative (group 5 versus group 6 in Tables 1 and 2). HBV-infected patients who did not develop anti-HBs included not only subjects who persistently showed positive $\mathrm{HBsAg}$ but also individuals with isolated antibodies to HBV core antigen (anti-HBc). The latter group showed neither $\mathrm{HBsAg}$ nor anti-HBs but presented anti-HBc positivity. This entire group of anti-HBs-negative subjects exposed to HBV infection showed lower IFN- $\lambda 3$ than patients who became anti-HBs positive after $\mathrm{HBV}$ infection. Significantly lower IFN- $\lambda 3$ concentrations in the mentioned groups were accompanied by significantly lower anti-HBs titres (groups 5 and 6 versus group 7 in Tables 1 and 2).

Among anti-HCV-positive subjects, circulating IFN- $\lambda 3$ was not significantly different between patients who resolved $\mathrm{HCV}$ infection and those who did not, independently of the subgroups being compared. All HCV RNA-negative patients developed higher anti-HBs titres than HCV RNA-positive subjects. The frequencies of responders and nonresponders to $\mathrm{HBV}$ vaccination, as well as subjects with anti-HBs titres $\geq 1000 \mathrm{IU} / \mathrm{L}$, were not significantly different between HCV RNA-negative and HCV RNA-positive individuals (group 9 versus group 10 in Tables 1 and 2). Similarly, HCV RNAnegative responders to $\mathrm{HBV}$ vaccination showed higher antiHBs titres than HCV RNA-positive responders (group 11 versus group 12 in Tables 1 and 2). Moreover, HCV RNAnegative responders showed higher anti-HBs than noninfected responders (group 11 versus group 2 in Tables 1 and 2). HCV RNA-positive responders, compared with noninfected responders, showed similar anti-HBs titres but lower IFN- $\lambda 3$ concentrations (group 12 versus group 2 in Tables 1 and 2).

In patients with both infections, $\mathrm{HBs} A g$-positive/HCV RNA-positive subjects showed lower IFN- $\lambda 3$ than patients who were positive only for HCV RNA (group 16 versus group 17 in Tables 1 and 2) or patients who resolved both infections (group 16 versus group 20 in Tables 1 and 2). Anti-HBs titres did not differ significantly between these groups.

The correlation coefficients between IFN- $\lambda 3$ and antiHBs titres in relevant subgroups of HD patients and healthy subjects exceeded 0.4 (Table 2).

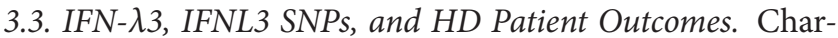
acteristics of HD patients with favourable/unfavourable outcomes with respect to $\mathrm{HBV}$ vaccination and occurrence of $\mathrm{HBV} / \mathrm{HCV}$ infections are shown in Table 3. Subjects with favourable outcomes showed higher circulating IFN$\lambda 3$, also after adjustment for age, duration of renal replacement therapy (RRT), low-flux HD, diabetic nephropathy, chronic glomerulonephritis, anuric status (daily urine output $\leq 100 \mathrm{~mL}$ ), alanine aminotransferase (ALT), and gammaglutamyltransferase (GGT). In the best multiple logistic regression model assessed using stepwise backward regression, IFN- $\lambda 3$ was the only positive determinant (OR 1.023, 


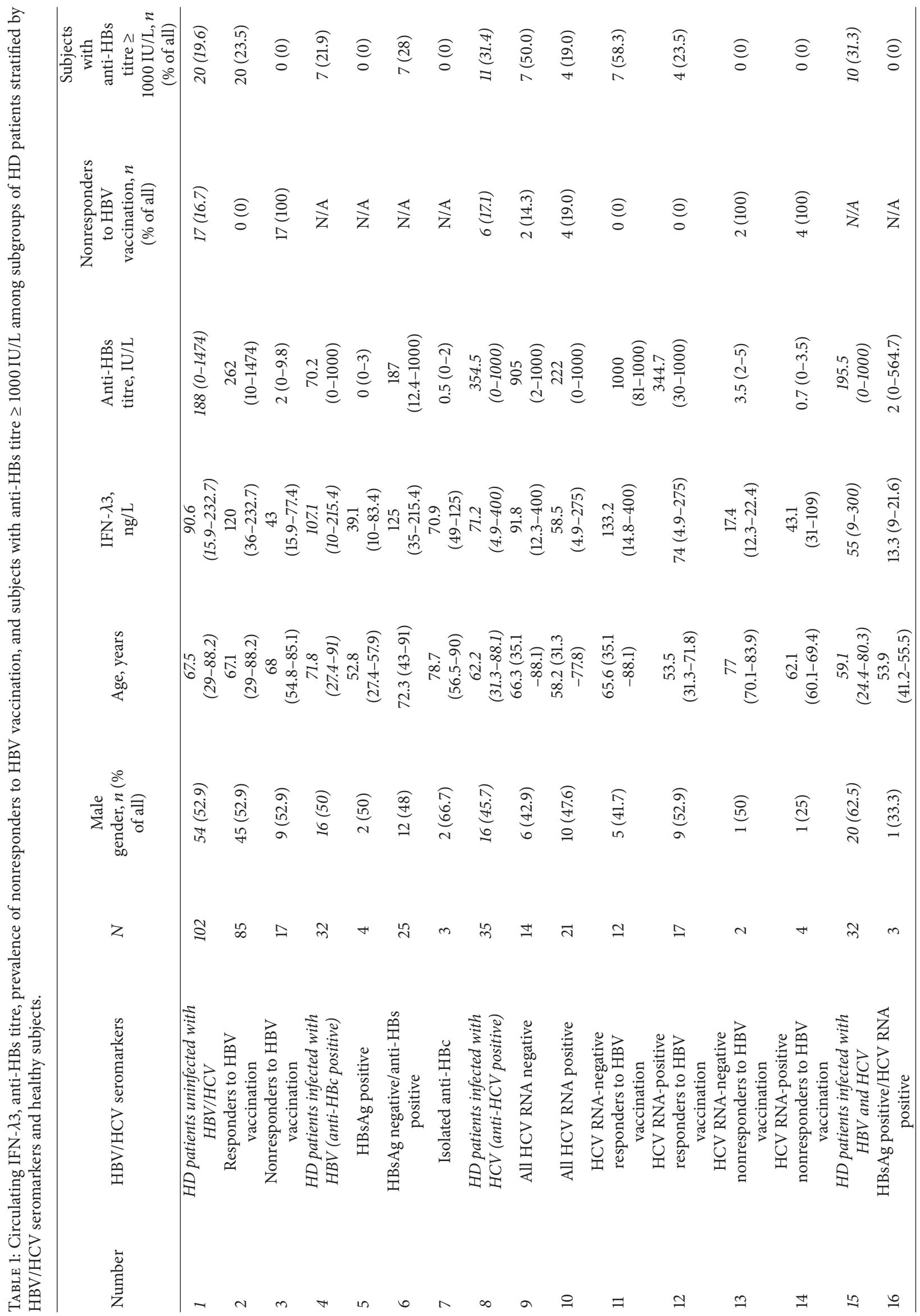




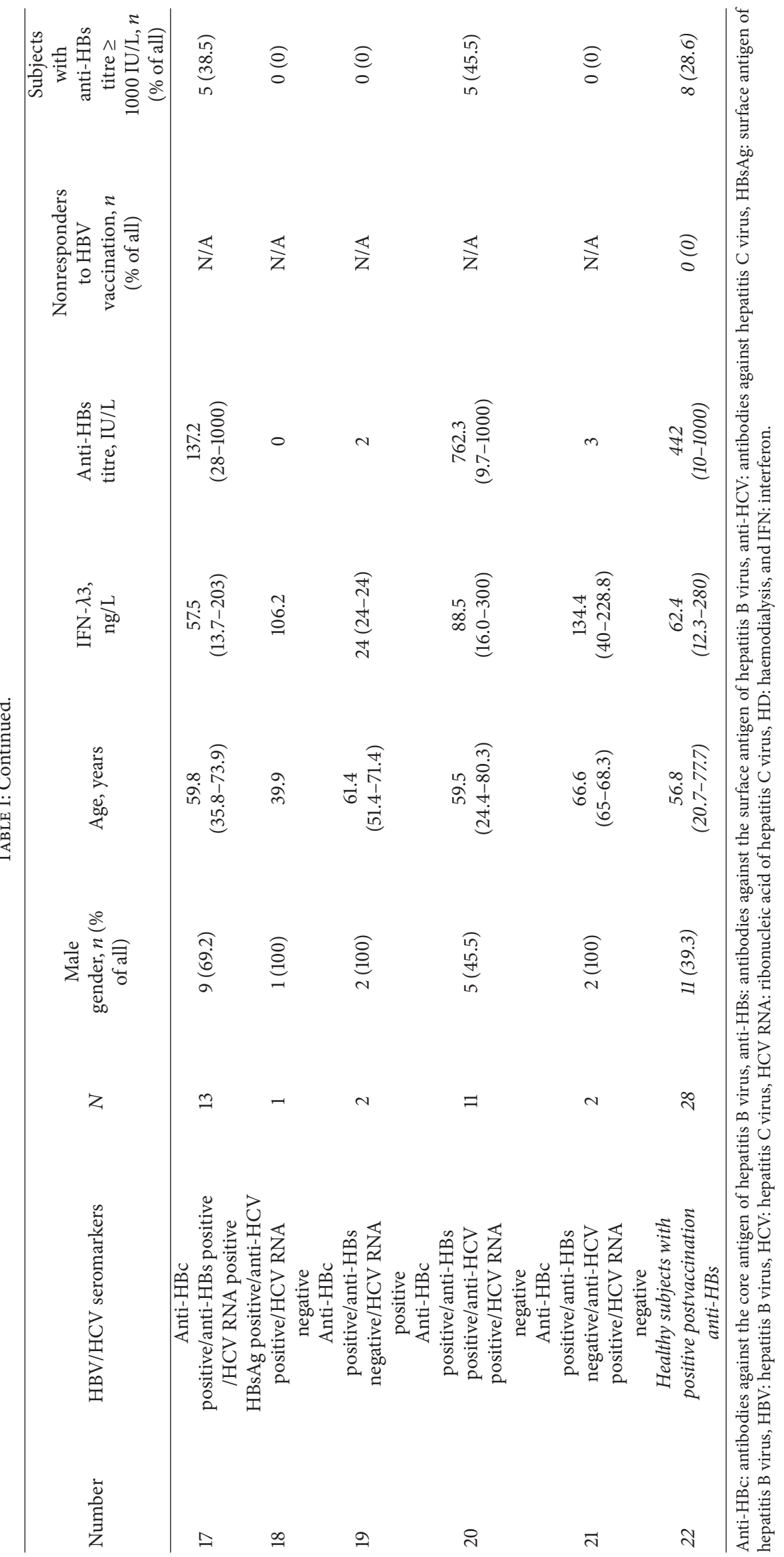




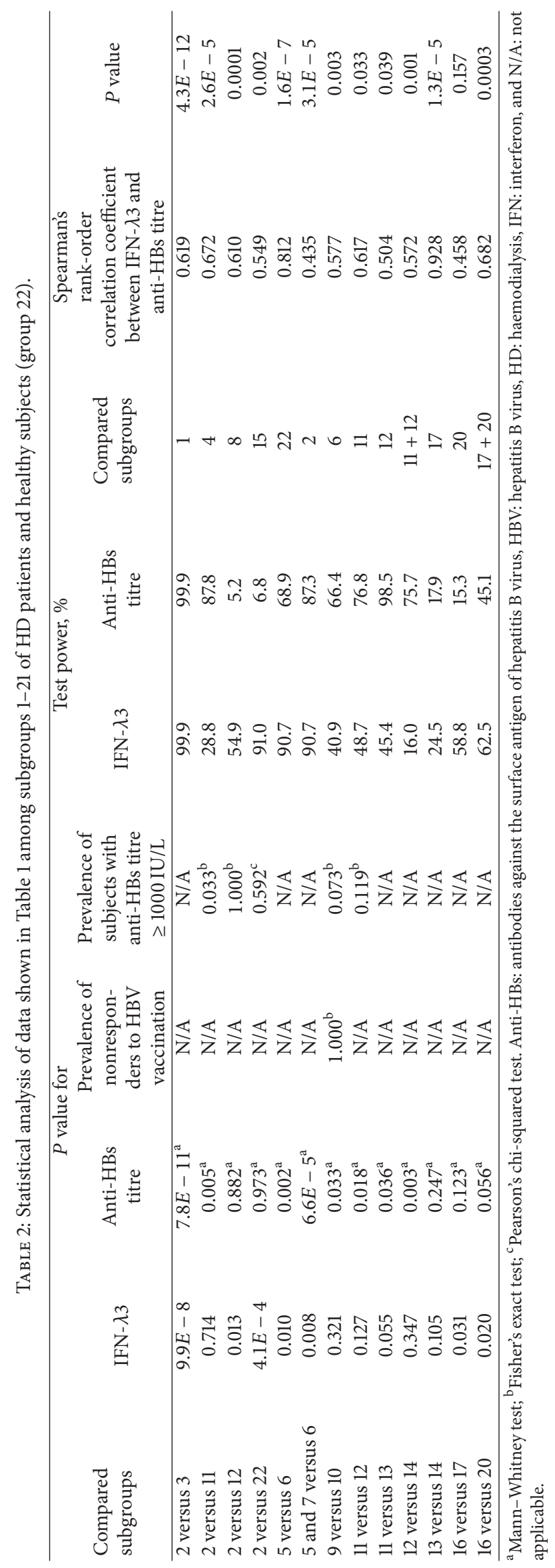


TABLE 3: Characteristics of HD patients showing unfavourable or favourable outcomes with respect to HBV vaccination and occurrence of $\mathrm{HBV} / \mathrm{HCV}$ infections.

\begin{tabular}{|c|c|c|c|}
\hline Parameter & $\begin{array}{c}\text { Unfavourable outcomes } \\
n=63 \\
(31 \% \text { of all }) \\
\end{array}$ & $\begin{array}{c}\text { Favourable outcomes } \\
n=138 \\
(69 \% \text { of all }) \\
\end{array}$ & $P$ value \\
\hline \multicolumn{4}{|l|}{ Demographic data } \\
\hline Male gender, $n, \%$ of all & $35(55.6)$ & $71(51.4)$ & $0.589^{\mathrm{a}}$ \\
\hline Age, years & $62.0(27.4-85)$ & $67.3(24.4-91)$ & $0.003^{\mathrm{c}}$ \\
\hline RRT duration, years & $7.2(0.1-30.2)$ & $4.9(0.2-23.1)$ & $0.030^{c}$ \\
\hline \multicolumn{4}{|l|}{ Cause of ESRD } \\
\hline Diabetic nephropathy, $n, \%$ of all & $11(17.5)$ & $46(33.3)$ & $0.021^{\mathrm{a}}$ \\
\hline Hypertensive nephropathy, $n$, $\%$ of all & $10(15.9)$ & $23(16.7)$ & $0.888^{\mathrm{a}}$ \\
\hline Chronic glomerulonephritis, $n$, $\%$ of all & $24(38.1)$ & $19(13.8)$ & $9.6 E-5^{\mathrm{a}}$ \\
\hline Chronic tubulointerstitial nephritis, $n, \%$ of all & $4(6.3)$ & $14(10.1)$ & $0.382^{\mathrm{a}}$ \\
\hline \multicolumn{4}{|l|}{ Type of RRT } \\
\hline Low-flux HD, $n$, \% of all & $40(63.5)$ & $70(50.7)$ & $0.092^{\mathrm{a}}$ \\
\hline High-flux HD, $n, \%$ of all & $21(33.3)$ & $64(46.4)$ & $0.082^{\mathrm{a}}$ \\
\hline $\mathrm{HDF}, n, \%$ of all & $2(3.2)$ & $4(2.9)$ & $1.000^{\mathrm{b}}$ \\
\hline PD as the first modality of RRT, $n, \%$ of all & $3(4.8)$ & $4(2.9)$ & $0.680^{\mathrm{b}}$ \\
\hline Daily urine output $\leq 100 \mathrm{~mL}, n, \%$ of all & $52(82.5)$ & $91(65.9)$ & $0.016^{\mathrm{a}}$ \\
\hline \multicolumn{4}{|l|}{ Laboratory data } \\
\hline IFN- $\lambda 3, \mathrm{ng} / \mathrm{L}$ & $50.7(4.9-275)$ & $120(14.8-400)$ & $1.4 E-11^{\mathrm{c}, \mathrm{d}}$ \\
\hline HBsAg positivity, $n, \%$ of all & $8(12.7)$ & $0(0)$ & $6.7 E-5^{\mathrm{b}}$ \\
\hline Anti-HBc positivity, $n, \%$ of all & $23(36.5)$ & $41(29.7)$ & $0.337^{\mathrm{a}}$ \\
\hline Anti-HBs titre, IU/L & $9.8(0-1000)$ & $299.3(0-1474)$ & $4.1 E-8^{c}$ \\
\hline Anti-HCV positivity, $n, \%$ of all & $42(66.7)$ & $25(18.1)$ & $1.3 E-11^{\mathrm{a}}$ \\
\hline HCV RNA positivity, $n, \%$ of all & $37(58.7)$ & $0(0)$ & $<2.2 E-16^{\mathrm{a}}$ \\
\hline $\mathrm{ALT}, \mathrm{IU} / \mathrm{L}$ & $18(4-90)$ & $13(1-57)$ & $0.005^{\mathrm{c}}$ \\
\hline AST, IU/L & $16(6-94)$ & $15(6-43)$ & $0.084^{\mathrm{c}}$ \\
\hline GGT, IU/L & $41(7-355)$ & $26(5-513)$ & $0.003^{c}$ \\
\hline ALP, U/L & $113.5(50.5-803.8)$ & $95.9(13.5-1353.3)$ & $0.132^{\mathrm{c}}$ \\
\hline C-reactive protein, $\mathrm{mg} / \mathrm{L}$ & $5.1(0.1-128.4)$ & $6.0(0-142)$ & $0.192^{\mathrm{c}}$ \\
\hline
\end{tabular}

${ }^{\mathrm{a}}$ Pearson's chi-squared test; ${ }^{\mathrm{b}}$ Fisher's exact test; ${ }^{\mathrm{c}}$ Mann-Whitney $U$ test; ${ }^{\mathrm{d}}$ the $P$ value after adjustment for age, RRT vintage, low-flux HD, diabetic nephropathy, chronic glomerulonephritis, urine output, ALT, and GGT using logistic regression was 6.7E-6. ALP: alkaline phosphatase, ALT: alanine aminotransferase, antiHBs: antibodies against the surface antigen of hepatitis B virus, anti-HBc: antibodies against the core antigen of hepatitis B virus, anti-HCV: antibodies against hepatitis C virus, AST: aspartate aminotransferase, ESRD: end-stage renal disease, GGT: gamma-glutamyltransferase, HBsAg: surface antigen of hepatitis B virus, HCV: hepatitis C virus, HD: haemodialysis, HDF: haemodiafiltration, IFN: interferon, N: number of patients, PD: peritoneal dialysis, RRT: renal replacement therapy.

95\% CI 1.013-1.033, $P=5.3 E-6)$, whereas chronic glomerulonephritis (OR $0.208,95 \%$ CI $0.079-0.544, P=0.001$ ) and ALT (OR 0.951, 95\% CI 0.913-0.990, $P=0.015$ ) were negative determinants of favourable outcome with respect to $\mathrm{HBV}$ vaccination and occurrence of $\mathrm{HBV} / \mathrm{HCV}$ infections. The AUC for this model equalled 0.863 .

Conversion factors to SI units are as follows: for alanine aminotransferase $1 \mathrm{U} / \mathrm{L}=0.0167 \mu \mathrm{kat} / \mathrm{L}$, for alkaline phosphatase $1 \mathrm{U} / \mathrm{L}=0.0167 \mu \mathrm{kat} / \mathrm{L}$, for aspartate aminotransferase $1 \mathrm{U} / \mathrm{L}=0.0167 \mu \mathrm{kat} / \mathrm{L}$, and for gamma-glutamyltransferase $1 \mathrm{U} / \mathrm{L}=0.0167 \mu \mathrm{kat} / \mathrm{L}$

In the entire HD group, there were no significant differences in circulating IFN- $\lambda 3$ with respect to IFNL3 SNPs (Table 4), and patients with favourable outcomes did not differ in distribution of IFNL3 SNPs compared with subjects with unfavourable outcomes, although some association was suggested by the $P_{\text {genotype }}$ (Table 5). It is noteworthy that associations between IFNL3 SNPs and spontaneous HCV clearance were observed in our study; however, because of the small number of HCV-infected patients, they were not significant after the Bonferroni correction $(P=0.018$ for CT + TT versus CC rs12979860 C > T, and $P=0.012$ for GG + GT versus TT rs8099917 T>G).

If low-flux HD patients were analysed separately from those treated with more efficient dialysis techniques (highflux HD and HDF), associations with both tested phenotypes (IFN- $\lambda 3$ and outcome) yielded better significance (Tables 6 and 7). However, when the Bonferroni correction was 
TABLE 4: Circulating IFN- $\lambda 3$ in relation to IFNL3 polymorphic variants in all HD patients.

\begin{tabular}{|c|c|c|c|c|c|}
\hline Tested SNP & $\begin{array}{c}\text { Major homozygote } \\
n \text { (frequency) } \\
\text { IFN- } \lambda 3, \mathrm{ng} / \mathrm{L}\end{array}$ & $\begin{array}{l}\text { Heterozygote } \\
n \text { (frequency) } \\
\text { IFN- } \lambda 3, \text { ng/L }\end{array}$ & $\begin{array}{c}\text { Minor homozygote } \\
n \text { (frequency) } \\
\text { IFN- } \lambda 3, \mathrm{ng} / \mathrm{L}\end{array}$ & Mode of inheritance & $P$ value \\
\hline \multirow{3}{*}{ IFNL3 rs8099917 } & TT & GT & GG & GG + GT versus TT & 0.136 \\
\hline & $101(0.51)$ & $89(0.45)$ & $10(0.05)$ & GG versus $\mathrm{GT}+\mathrm{TT}$ & 0.327 \\
\hline & $79.9(10-264)$ & $109(4.9-400)$ & $66(13.7-207)$ & TT versus GG & 0.523 \\
\hline \multirow{3}{*}{ IFNL3 rs12979860 } & $\mathrm{CC}$ & $\mathrm{CT}$ & $\mathrm{TT}$ & $\mathrm{TT}+\mathrm{CT}$ versus $\mathrm{CC}$ & 0.606 \\
\hline & $67(0.34)$ & $83(0.42)$ & $50(0.25)$ & $\mathrm{TT}$ versus $\mathrm{CT}+\mathrm{CC}$ & 0.064 \\
\hline & $79(10-264)$ & $86(4.9-400)$ & $98.6(9-215.4)$ & CC versus TT & 0.100 \\
\hline
\end{tabular}

TABLE 5: IFNL3 polymorphic variants in HD patients showing different outcomes with respect to HBV vaccination and $\mathrm{HBV} / \mathrm{HCV}$ infections.

\begin{tabular}{|c|c|c|c|c|c|c|}
\hline & $\begin{array}{c}\text { Unfavourable } \\
\text { outcomes } \\
\text { (n, frequency) } \\
n=62\end{array}$ & $\begin{array}{c}\text { Favourable } \\
\text { outcomes } \\
\text { (n, frequency) } \\
n=138\end{array}$ & Odds ratio $(95 \% \mathrm{CI})$ & $P$ value $^{\mathrm{a}}$ & $P_{\text {trend }}{ }^{\mathrm{b}}$ & $P_{\text {genotype }}{ }^{\mathrm{a}}$ \\
\hline \multicolumn{7}{|c|}{ IFNL3 rs12979860 } \\
\hline $\mathrm{CC}$ & $18(29)$ & $49(35.5)$ & Reference & - & 0.583 & 0.027 \\
\hline $\mathrm{CT}$ & $34(54.8)$ & $49(35.5)$ & $1.889(0.943-3.785)$ & 0.071 & & \\
\hline $\mathrm{TT}$ & $10(16.1)$ & $40(29.0)$ & $0.681(0.283-1.639)$ & 0.389 & & \\
\hline $\mathrm{CT}+\mathrm{TT}$ versus $\mathrm{CC}$ & $44(71.0)$ & $89(64.5)$ & $1.346(0.703-2.578)$ & 0.370 & & \\
\hline TT versus CC + CT & $10(16.1)$ & $40(29.0)$ & $0.471(0.218-1.018)$ & 0.052 & & \\
\hline MAF & $(0.44)$ & $(0.47)$ & $0.879(0.574-1.347)$ & 0.554 & & \\
\hline \multicolumn{7}{|c|}{ IFNL3 rs8099917 } \\
\hline TT & $30(48.4)$ & $71(51.4)$ & Reference & - & 0.754 & $0.933^{c}$ \\
\hline GT & $29(46.8)$ & $60(43.5)$ & $1.144(0.618-2.117)$ & 0.668 & & \\
\hline GG & $3(4.8)$ & $7(5.1)$ & $1.014(0.246-4.189)$ & $1.000^{\mathrm{c}}$ & & \\
\hline $\mathrm{GG}+\mathrm{GT}$ versus $\mathrm{TT}$ & $32(51.6)$ & $67(48.6)$ & $1.130(0.621-2.059)$ & 0.689 & & \\
\hline GG versus GT + TT & $3(4.8)$ & $7(5.1)$ & $0.952(0.238-3.809)$ & $1.000^{\mathrm{c}}$ & & \\
\hline MAF & $(0.28)$ & $(0.27)$ & $1.073(0.669-1.723)$ & 0.769 & & \\
\hline
\end{tabular}

MAF: minor allele frequency. ${ }^{\mathrm{a}}$ Pearson's chi-squared test; ${ }^{\mathrm{b}}$ Cochran-Armitage trend test; ${ }^{\mathrm{c}}$ Fisher's exact test.

applied, significance was no longer detected. Such differences were not shown among patients treated with high-flux HD or those treated with HDF.

IFNL3 haplotype frequencies were not different between patients showing favourable and unfavourable outcomes (all $P$ values $>0.27)$. There were also no differences when haplotypes were compared between outcomes separately among patients treated with low-flux HD (all $P$ values $>0.21$ ) or highflux HD and HDF (all $P$ values $>0.21$ ).

In univariate analyses, circulating IFN- $\lambda 3$ correlated positively with anti-HBs titre and negatively with duration of RRT, anuric status, HCV RNA positivity, and HBsAg positivity (Table 8 ). Variables associated with IFN- $\lambda 3$ concentrations in univariate analyses were included in the multiple linear regression analysis. After stepwise backward selection, the best model showed anti-HBs titre, HCV RNA positivity, and HBsAg positivity as independent explanatory correlates of circulating IFN- $\lambda 3$ (multiple $R$ squared for the model: 0.293 , adjusted $R$ squared for the model: $0.282, P$ value: $9.4 E-15$ ).
To show the values of the tested variables with respect to circulating IFN- $\lambda 3$ concentration, we compared patient characteristics after stratification of all patients according to tertiles of IFN- $\lambda 3$ level (Table 9). The results of this approach showed that patients with IFN- $\lambda 3$ levels in the lower tertile demonstrated higher prevalence of positive tests for $\mathrm{HBsAg}$ and anti-HCV, a lower anti-HBs titre, and a lower frequency of favourable outcomes than patients in the upper tertile of IFN- $\lambda 3$ levels.

Conversion factors to SI units are as follows: for alanine aminotransferase $1 \mathrm{U} / \mathrm{L}=0.0167 \mu \mathrm{kat} / \mathrm{L}$, for alkaline phosphatase $1 \mathrm{U} / \mathrm{L}=0.0167 \mu \mathrm{kat} / \mathrm{L}$, for aspartate aminotransferase $1 \mathrm{U} / \mathrm{L}=0.0167 \mu \mathrm{kat} / \mathrm{L}$, and for gamma-glutamyltransferase $1 \mathrm{U} / \mathrm{L}=0.0167 \mu \mathrm{kat} / \mathrm{L}$.

\section{Discussion}

Comparison of responders to $\mathrm{HBV}$ vaccination among $\mathrm{HD}$ patients free of $\mathrm{HBV} / \mathrm{HCV}$ infections and healthy subjects, 
TABLE 6: Circulating IFN- $\lambda 3$ in relation to IFNL3 polymorphisms in patients treated with low-flux HD.

\begin{tabular}{|c|c|c|c|c|c|}
\hline Tested SNP & $\begin{array}{c}\text { Major homozygote } \\
n \text { (frequency) } \\
\text { IFN- } \lambda 3, \mathrm{ng} / \mathrm{L} \\
\end{array}$ & $\begin{array}{l}\text { Heterozygote } \\
n \text { (frequency) } \\
\text { IFN- } \lambda 3, \mathrm{ng} / \mathrm{L}\end{array}$ & $\begin{array}{c}\text { Minor homozygote } \\
n \text { (frequency) } \\
\text { IFN- } \lambda 3, \mathrm{ng} / \mathrm{L}\end{array}$ & Mode of inheritance & $P$ value \\
\hline \multirow{3}{*}{ IFNL3 rs8099917 } & $\mathrm{TT}$ & GT & GG & $\mathrm{GG}+\mathrm{GT}$ versus $\mathrm{TT}$ & 0.365 \\
\hline & $55(0.50)$ & $48(0.44)$ & $7(0.06)$ & GG versus GT + TT & 0.845 \\
\hline & $87.3(10-264)$ & $109.2(9-400)$ & $74(48.5-207)$ & TT versus GG & 1.000 \\
\hline \multirow{3}{*}{ IFNL3 rs12979860 } & $\mathrm{CC}$ & $\mathrm{CT}$ & $\mathrm{TT}$ & $\mathrm{TT}+\mathrm{CT}$ versus $\mathrm{CC}$ & 0.770 \\
\hline & $31(0.28)$ & $44(0.40)$ & $35(0.32)$ & $\mathrm{TT}$ versus $\mathrm{CT}+\mathrm{CC}$ & $0.027^{\mathrm{a}}$ \\
\hline & $95.6(10-264)$ & $80.8(16-400)$ & $115.1(9-207)$ & CC versus TT & 0.188 \\
\hline
\end{tabular}

${ }^{a}$ Not significant after the Bonferroni correction $(P>0.004)$.

TABLE 7: IFNL3 polymorphic variants in HD patients treated with low-flux HD showing different outcomes with respect to HBV vaccination and $\mathrm{HBV} / \mathrm{HCV}$ infections.

\begin{tabular}{|c|c|c|c|c|c|c|}
\hline & $\begin{array}{c}\text { Unfavourable } \\
\text { outcomes } \\
\text { (n, frequency) } \\
n=40\end{array}$ & $\begin{array}{c}\text { Favourable } \\
\text { outcomes } \\
\text { (n, frequency) } \\
n=70\end{array}$ & Odds ratio $(95 \% \mathrm{CI})$ & $P$ value $^{\mathrm{a}}$ & $P_{\text {trend }}^{\mathrm{b}}$ & $P_{\text {genotype }}{ }^{\mathrm{a}}$ \\
\hline \multicolumn{7}{|c|}{ IFNL3 rs12979860 } \\
\hline $\mathrm{CC}$ & $10(25)$ & $21(30)$ & Reference & - & 0.254 & $0.011^{c}$ \\
\hline $\mathrm{CT}$ & $23(57.5)$ & $21(30)$ & $2.300(0.883-5.993)$ & 0.086 & & \\
\hline $\mathrm{TT}$ & $7(17.5)$ & $28(40)$ & $0.525(0.171-1.608)$ & 0.256 & & \\
\hline $\begin{array}{l}\mathrm{CT}+\mathrm{TT} \text { versus } \\
\mathrm{CC}\end{array}$ & $30(75)$ & $49(70)$ & $1.286(0.534-3.098)$ & 0.575 & & \\
\hline $\begin{array}{l}\mathrm{TT} \text { versus } \mathrm{CC}+ \\
\mathrm{CT}\end{array}$ & $7(17.5)$ & $28(40)$ & $0.318(0.124-0.819)$ & $0.015^{\mathrm{c}}$ & & \\
\hline MAF & $(0.46)$ & $(0.55)$ & $0.704(0.406-1.222)$ & 0.212 & & \\
\hline \multicolumn{7}{|c|}{ IFNL3 rs8099917 } \\
\hline $\mathrm{TT}$ & $20(50)$ & $35(50)$ & Reference & - & 0.616 & $0.519^{\mathrm{d}}$ \\
\hline GT & $19(47.5)$ & $29(41.4)$ & $1.147(0.516-2.546)$ & 0.737 & & \\
\hline GG & $1(2.5)$ & $6(8.6)$ & $0.292(0.033-2.599)$ & $0.406^{\mathrm{d}}$ & & \\
\hline $\begin{array}{l}\text { GG + GT versus } \\
\text { TT }\end{array}$ & $20(50)$ & $35(50)$ & $1.000(0.460-2.175)$ & 1.000 & & \\
\hline $\begin{array}{l}\text { GG versus } \mathrm{GT}+ \\
\mathrm{TT}\end{array}$ & $1(2.5)$ & $6(8.6)$ & $0.274(0.032-2.358)$ & $0.419^{\mathrm{d}}$ & & \\
\hline MAF & $(0.26)$ & $(0.29)$ & $0.859(0.464-1.593)$ & 0.630 & & \\
\hline
\end{tabular}

MAF: minor allele frequency. ${ }^{\mathrm{a}}$ Pearson's chi-squared test; ${ }^{\mathrm{b}}$ Cochran-Armitage trend test; ${ }^{\mathrm{c}}$ not significant after the Bonferroni correction $(P>0.004) ;{ }^{\mathrm{d}}$ Fisher's exact test IFN- $\lambda 3$ correlates.

all being responders to $\mathrm{HBV}$ vaccination, showed that IFN$\lambda 3$ is upregulated in HD patients. In uremic rats, 2665 of 10,153 genes were differentially expressed, with $47 \%$ up- and $53 \%$ downregulated [23]. IFN- $\lambda 3$ may be upregulated in a uremic milieu; however, a lower degradation rate is also a possibility, as with many other biologically active proteins [24-27]. In our study, anuric status (daily urine output $\leq$ $100 \mathrm{~mL}$ ) seemed to negatively influence IFN- $\lambda 3$ concentrations, but this correlation was not statistically significant after adjustment for the variables that were significant in univariate analyses. Moreover, circulating IFN- $\lambda 3$ levels did not correlate with urine output in HD patients with preserved residual renal function and anuric patients did not differ in IFN- $\lambda 3$ concentrations from those with daily diuresis over $100 \mathrm{~mL}$ (data not shown). This evidence suggests no relationship between IFN- $\lambda 3$ levels and urine output in HD patients.

Circulating IFN- $\lambda 3$ concentrations are shown to be associated with response to HBV vaccination [16] as well as with resolution of HBV [15] and HCV [14] infections. We investigated associations between plasma IFN- $\lambda 3$ and anti$\mathrm{HBs}$ titres in $\mathrm{HD}$ patients not infected with $\mathrm{HBV} / \mathrm{HCV}$, infected with either HBV or HCV alone, and exposed to both HBV and HCV.

In $\mathrm{HBV} / \mathrm{HCV}$-noninfected responders to $\mathrm{HBV}$ vaccination among $\mathrm{HD}$ patients, there was a significant positive correlation between IFN- $\lambda 3$ and anti-HBs titres, as in healthy responders. It is not clear whether there is a direct causal 
TABLE 8: Explanatory and response correlates of the circulating IFN- $\lambda 3$ concentration among demographic, clinical, and laboratory data of the entire group of HD patients $(n=201)$.

\begin{tabular}{|c|c|c|c|c|}
\hline \multirow{2}{*}{ Parameter } & \multicolumn{2}{|c|}{ Unadjusted } & \multicolumn{2}{|c|}{ Adjusted $^{\mathrm{b}}$} \\
\hline & $\beta^{\mathrm{a}} \pm \mathrm{SE}$ & $P$ value & $\beta^{\mathrm{a}} \pm \mathrm{SE}$ & $P$ value \\
\hline Male gender & $-10 \pm 10.4$ & 0.340 & $0.7 \pm 9.0$ & 0.939 \\
\hline Age (per 10 years) & $-1.9 \pm 3.5$ & 0.581 & $-3.5 \pm 3.2$ & 0.288 \\
\hline RRT vintage (per 1 year) & $-1.7 \pm 0.8$ & 0.037 & $-0.2 \pm 0.9$ & 0.861 \\
\hline Diabetic nephropathy & $1.6 \pm 11.6$ & 0.892 & $-9.4 \pm 10.0$ & 0.347 \\
\hline Hypertensive nephropathy & $3.1 \pm 14.1$ & 0.825 & $4.5 \pm 12.1$ & 0.710 \\
\hline Chronic glomerulonephritis & $-6.2 \pm 12.7$ & 0.629 & $9.8 \pm 11.8$ & 0.407 \\
\hline Chronic tubulointerstitial nephritis & $18 \pm 18.3$ & 0.327 & $8.9 \pm 15.6$ & 0.569 \\
\hline LF-HD & $7.3 \pm 10.5$ & 0.489 & $11.1 \pm 9.0$ & 0.222 \\
\hline PD as the first modality of RRT & $-17.2 \pm 28.5$ & 0.546 & $23.6 \pm 25.0$ & 0.347 \\
\hline Daily urine output $\leq 100 \mathrm{~mL}$ & $-24.8 \pm 11.4$ & 0.030 & $-13.1 \pm 10.8$ & 0.230 \\
\hline HBsAg positivity & $-71.3 \pm 26.2$ & 0.007 & $-41.9 \pm 23.8$ & 0.079 \\
\hline Anti-HBc positivity & $8.7 \pm 11.2$ & 0.439 & $1.5 \pm 10.2$ & 0.880 \\
\hline Anti-HCV positivity & $-15 \pm 11$ & 0.177 & $-5.7 \pm 13.5$ & 0.675 \\
\hline Anti-HBs titre (per $100 \mathrm{IU} / \mathrm{L}$ ) & $8.9 \pm 1.1$ & $3.6 E-14$ & $8.4 \pm 1.1$ & $6.4 E-13$ \\
\hline HCV RNA positivity & $-36.7 \pm 13.2$ & 0.006 & $-28.5 \pm 12.9$ & 0.028 \\
\hline ALT (per 1 IU/L) & $-0.8 \pm 0.4$ & 0.066 & $-0.1 \pm 0.4$ & 0.780 \\
\hline AST (per 1IU/L) & $-0.4 \pm 0.5$ & 0.423 & $0.2 \pm 0.4$ & 0.701 \\
\hline GGT (per 1 IU/L) & $-0.03 \pm 0.08$ & 0.713 & $0.06 \pm 0.07$ & 0.426 \\
\hline ALP (per 1 IU/L) & $-0.03 \pm 0.03$ & 0.385 & $-0.02 \pm 0.03$ & 0.438 \\
\hline C-reactive protein (per $1 \mathrm{mg} / \mathrm{L}$ ) & $-0.06 \pm 0.30$ & 0.831 & $-0.005 \pm 0.256$ & 0.985 \\
\hline
\end{tabular}

$\beta$ coefficient and SE values can be interpreted as follows: for a unitary change in an analysed parameter, the circulating IFN- $\lambda 3$ concentration would change by $\beta \pm \mathrm{SE}$ (ng/L). ${ }^{\mathrm{b}}$ Adjusted for RRT vintage, anti-HBs titre, HBsAg positivity, HCV RNA positivity, and urine output, as appropriate. ALP: alkaline phosphatase, ALT: alanine aminotransferase, anti-HBc: antibodies against the core antigen of hepatitis B virus, anti-HBs: antibodies against the surface antigen of hepatitis B virus, anti-HCV: antibodies against hepatitis C virus, AST: aspartate aminotransferase, GGT: gamma-glutamyltransferase, HBsAg: surface antigen of hepatitis B virus, HCV RNA: ribonucleic acid of hepatitis C virus, HD: haemodialysis, IFN: interferon, LF-HD: low-flux haemodialysis, PD: peritoneal dialysis, and RRT: renal replacement therapy.

association between IFN- $\lambda 3$ and anti-HBs formation after vaccination. Indoleamine 2,3-dioxygenase (IDO) might be involved. IDO gene activity is directly induced by IFN $-\lambda 3$ in a dose-dependent manner [28]. Therefore, more efficient antiHBs production in the presence of higher circulating IFN$\lambda 3$ levels might occur due to IFN- $\lambda 3$-induced upregulation of IDO expression. A mouse study showing that IDO inhibition at the time of vaccination with HBsAg decreased anti-HBs titre is in accordance with this concept [29]. Additionally, increased IDO activity seems to skew T helper (Th) cell polarization towards a Th2 pathway, which primarily results in antibody formation $[30,31]$. The addition of IFN $-\lambda 3$ to immunizations in mice caused a greater than 2 -fold increase in the titres of IgG2a antigen-specific antibodies produced after Thl pathway stimulation. An increased release of the Th1-associated cytokine IFN- $\gamma$ was also observed [32]. In HD patients, a deficit in IFN $-\gamma$ was noted despite the increased blood levels of Thl cytokines involved in IFN- $\gamma$ production, such as IL-18 [33]. Moreover, when used as an adjuvant during vaccination against a lethal influenza virus in mice, IFN- $\lambda 3$ induced $100 \%$ protection from mortality associated with this viral infection [32]. However, these results were not confirmed in rhesus macaques when the generation of IgG2a antigen-specific antibodies was examined following the addition of IFN- $\lambda 3$ to the immunization strategy [34]. It remains unknown how IFN- $\lambda 3$ is stimulated by immunizations with HBsAg.

A positive correlation of IFN- $\lambda 3$ with anti-HBs titre was also demonstrated in HD patients who were able to generate anti-HBs in response to HBV infection. We postulate that HBV might be a trigger for IFN- $\lambda 3$ production and that rising IFN- $\lambda 3$ concentrations promote anti-HBs development. A study by Sato et al. [35] demonstrated that type III IFNs, including IFN- $\lambda 3$, are induced in human hepatocytes during HBV infection. Retinoic acid-inducible gene 1 (RIG-1) senses the HBV genotypes $\mathrm{A}, \mathrm{B}$, and $\mathrm{C}$ for the induction of type III IFNs through its recognition of the $5^{\prime}-\varepsilon$ region of HBVderived pregenomic RNA. In primary human hepatocytes, significant $\mathrm{HBV}$-related induction was observed for IFN- $\lambda 1$, IFN- $\lambda 2$, and IFN- $\lambda 3$ mRNAs. Moreover, after chimeric mice were intravenously infected with HBV genotype $C$ derived from chronic hepatitis B patients, the expression of IFN$\lambda 1$, IFN- $\lambda 2$, and IFN- $\lambda 3$ mRNAs in the liver tissue was found to be increased at 4 or 5 weeks after infection [35]. Additionally, IDO elevations were observed in acute hepatitis B patients with self-limited HBV infection [36]. After HBV infection, HBsAg clearance is usually associated with the development of anti-HBs, although the anti-HBs response 
TABLE 9: Characteristics of HD patients stratified by tertiles of circulating IFN- $\lambda 3$ concentration.

\begin{tabular}{|c|c|c|c|c|c|}
\hline \multirow[b]{2}{*}{ Parameter } & \multicolumn{3}{|c|}{ Tertiles of circulating IFN- $\lambda 3$ concentration } & \multirow[b]{2}{*}{$P$ value } & \multirow[b]{2}{*}{$\begin{array}{l}P \text { for trend } \\
\text { test }\end{array}$} \\
\hline & $\begin{array}{c}\text { Lower }(\leq 64 \mathrm{ng} / \mathrm{L}) \\
\qquad N=67\end{array}$ & $\begin{array}{c}\text { Middle } \\
(65-123 \mathrm{ng} / \mathrm{L}) \\
N=68\end{array}$ & $\begin{array}{c}\text { Upper }(\geq 124 \mathrm{ng} / \mathrm{L}) \\
N=66\end{array}$ & & \\
\hline \multicolumn{6}{|l|}{ Demographic data } \\
\hline Male gender, $n, \%$ of all & $39(58.2)$ & $36(52.9)$ & $31(47.0)$ & $0.430^{\mathrm{a}}$ & 0.194 \\
\hline Age, years & $64.9(27.4-88.1)$ & $66.2(29.5-88.2)$ & $66.4(24.4-91)$ & $0.840^{\mathrm{b}}$ & \\
\hline RRT duration, years & $5.6(0.1-29.9)$ & $5.2(0.2-30.2)$ & $5(0.3-26.5)$ & $0.722^{\mathrm{b}}$ & \\
\hline \multicolumn{6}{|l|}{ Cause of ESRD } \\
\hline $\begin{array}{l}\text { Diabetic nephropathy, } n \text {, \% of } \\
\text { all }\end{array}$ & $21(31.3)$ & $16(23.5)$ & $22(30.3)$ & $0.418^{\mathrm{a}}$ & 0.805 \\
\hline $\begin{array}{l}\text { Hypertensive nephropathy, } n \text {, } \\
\% \text { of all }\end{array}$ & $11(16.4)$ & $11(16.2)$ & $11(16.7)$ & $0.997^{\mathrm{a}}$ & 0.969 \\
\hline $\begin{array}{l}\text { Chronic glomerulonephritis, } \\
n, \% \text { of all }\end{array}$ & $13(19.4)$ & $18(26.5)$ & $12(18.2)$ & $0.448^{\mathrm{a}}$ & 0.868 \\
\hline $\begin{array}{l}\text { Chronic tubulointerstitial } \\
\text { nephritis, } n, \% \text { of all }\end{array}$ & $8(11.9)$ & $2(2.9)$ & $8(12.2)$ & $0.102^{\mathrm{a}}$ & 0.978 \\
\hline \multicolumn{6}{|l|}{ Type of $R R T$} \\
\hline Low-flux HD, $n$, $\%$ of all & $34(50.7)$ & $35(51.5)$ & $41(62.1)$ & $0.337^{\mathrm{a}}$ & 0.489 \\
\hline High-flux HD, $n$, \% of all & $31(46.3)$ & $29(42.6)$ & $25(37.9)$ & $0.617^{\mathrm{a}}$ & 0.328 \\
\hline HDF, $n, \%$ of all & $2(3.0)$ & $4(5.9)$ & $0(0)$ & $0.169^{c}$ & 0.315 \\
\hline $\begin{array}{l}\text { PD as the first modality of } \\
\text { RRT, } n, \% \text { of all }\end{array}$ & $3(4.5)$ & $2(2.9)$ & $2(3.0)$ & $0.900^{c}$ & 0.648 \\
\hline $\begin{array}{l}\text { Daily urine output } \leq 100 \mathrm{~mL} \text {, } \\
n, \% \text { of all }\end{array}$ & $50(74.6)$ & $53(77.9)$ & $40(60.6)$ & $0.064^{\mathrm{a}}$ & 0.075 \\
\hline \multicolumn{6}{|l|}{ Laboratory data } \\
\hline HBsAg positivity, $n, \%$ of all & $6(9.0)$ & $2(2.9)$ & $0(0)$ & $0.021^{\mathrm{c}}$ & 0.008 \\
\hline Anti-HBc positivity, $n, \%$ of all & $24(35.8)$ & $17(25)$ & $23(34.8)$ & $0.328^{\mathrm{a}}$ & 0.899 \\
\hline Anti-HBs titre, IU/L & $26.3(0-1000)$ & $192(0-1474)$ & $960.3(0-1000)$ & $1.9 E-13^{\mathrm{b}}$ & \\
\hline $\begin{array}{l}\text { Anti-HCV positivity, } n, \% \text { of } \\
\text { all }\end{array}$ & $33(49.3)$ & $16(23.5)$ & $18(27.3)$ & $0.003^{\mathrm{a}}$ & 0.007 \\
\hline $\begin{array}{l}\text { HCV RNA positivity, } n, \% \text { of } \\
\text { all }\end{array}$ & $20(60.6)$ & $11(16.2)$ & $6(33.3)$ & $0.080^{\mathrm{a}}$ & 0.096 \\
\hline ALT, IU/L & $15(3-90)$ & $14(3-52)$ & $12(1-57)$ & $0.106^{\mathrm{b}}$ & \\
\hline AST, IU/L & $15(6-94)$ & $15(7-68)$ & $15.5(6-43)$ & $0.750^{\mathrm{b}}$ & \\
\hline GGT, IU/L & $35(7-213)$ & $29.5(5-441)$ & $24(10-513)$ & $0.202^{\mathrm{b}}$ & \\
\hline ALP, U/L & $99.8(54.3-1109.5)$ & $98.5(41-1299.3)$ & $105.9(13.5-1353.3)$ & $0.808^{\mathrm{b}}$ & \\
\hline C-reactive protein, $\mathrm{mg} / \mathrm{L}$ & $5.2(0.1-128.4)$ & $7.3(0.5-105.5)$ & $5.1(0-142)$ & $0.187^{\mathrm{b}}$ & \\
\hline Favourable outcomes & $26(38.8)$ & $52(76.5)$ & $60(90.9)$ & $1.8 E-10^{\mathrm{a}}$ & $8.9 E-11$ \\
\hline
\end{tabular}

alone cannot account for HBsAg clearance [37, 38]. In a nonuremic Han Chinese population, serum IFN- $\lambda 3$ levels were lower in patients with chronic HBV infection than in subjects with self-limited HBV infection or healthy subjects. A gene expression microarray analysis showed enhanced IFN- $\lambda 3$ expression in patients with a low HBV viral load [15]. In our previous study [16], HBV-infected patients who had severe renal damage requiring HD treatment and were not able to generate anti-HBs included subjects with chronic hepatitis B and subjects with isolated anti-HBc positivity. These combined groups showed lower IFN- $\lambda 3$ than subjects who developed anti-HBs after infection. This finding was also relevant in the current study. Moreover, IFN- $\lambda 3$ was a significant independent predictor of HBsAg clearance in 
the HD group. Therefore, the current study demonstrates that, in HD patients, higher circulating IFN- $\lambda 3$ levels may be associated with self-limited HBV infection.

An endogenous IFN response is activated during $\mathrm{HCV}$ infection; however, IFN induction, IFN signalling, and transcription of IFN-stimulated gene mRNA are not efficient in hepatocytes from chronic hepatitis C patients [39]. Circulating IFN- $\lambda 3$ levels may be associated with the outcome of HCV infection. In a study by Shi et al. [18], patients with persistent $\mathrm{HCV}$ infection (median viral load 11.2E5 IU/mL) showed lower circulating IFN- $\lambda 3$ than patients who cleared their infections or healthy controls. Subjects who spontaneously cleared HCV revealed similar circulating IFN- $\lambda 3$ levels to a healthy group [40]. In a study by Aoki et al. [41], chronic hepatitis C patients demonstrated substantially higher IFN- $\lambda 3$ levels than healthy volunteers. After treatment with pegylated IFN- $\alpha$ and ribavirin, the increased IFN- $\lambda 3$ levels returned to normal values in patients who cleared their $\mathrm{HCV}$, but treatment effects were not related to baseline circulating IFN- $\lambda 3$ levels [41]. In these two aforementioned studies, renal function was not mentioned and was probably normal. Similarly, there were no data on HBV vaccination in these studied groups. In our study, there were no significant differences in circulating IFN- $\lambda 3$ between patients with spontaneous $\mathrm{HCV}$ resolution and $\mathrm{HCV}$-infected patients when all patients, responsive or unresponsive to $\mathrm{HBV}$ vaccination, were compared within HCV-exposed subgroups. However, although not significantly different (perhaps as a result of small sample sizes), differences in IFN- $\lambda 3$ suggested lower IFN- $\lambda 3$ in all HCV RNA-positive patients and in HCV RNA-positive responders compared with HCV RNAnegative subjects. Moreover, responders to $\mathrm{HBV}$ vaccination with persistent $\mathrm{HCV}$ infection showed lower IFN- $\lambda 3$ than infection-free responders, whereas responders who resolved $\mathrm{HCV}$ infection showed similar IFN- $\lambda 3$ levels to infectionfree responders. The latter group may represent a "control" status among HD subjects, given that all patients were free from $\mathrm{HBV} / \mathrm{HCV}$ infections and were able to respond to $\mathrm{HBV}$ vaccination, which is a predictor of longer survival in HD population [42]. Additionally, IFN- $\lambda 3$ was a significant independent negative explanatory variable for HCV RNA positivity in the entire HD group, which argues for an association between higher IFN- $\lambda 3$ and spontaneous HCV resolution. Therefore, our results are more consistent with those of Shi et al. [18]. However, patients examined by Aoki et al. [41] probably showed more severe HCV disease course (all had a diagnosis of chronic hepatitis $\mathrm{C}$, the group showed a high mean viral load of $2.0 E 6 \mathrm{IU} / \mathrm{mL}$, and all patients required antiviral therapy), whereas our patients were diagnosed as long-term HCV carriers with lower viral load and were not qualified for IFN-based therapy. This patient-related difference may be important for IFN- $\lambda 3$ levels. In accordance with this discrepancy are data showing that IFNL3 alleles favourable for spontaneous HCV resolution may be associated with increased inflammation and higher fibrosis scores in HCV-infected subjects [40].

Among population with normal renal function, responsiveness to $\mathrm{HBV}$ vaccination is lower in subjects infected with $\mathrm{HCV}$, whereas individuals who cleared HCV show higher vaccine effectiveness than subjects not exposed to $\mathrm{HCV}$ [43]. In the studied HD patients, responsiveness to $\mathrm{HBV}$ vaccination was similar in noninfected and $\mathrm{HCV}$ infected patients, but $\mathrm{HBV}$ vaccine responders who did not resolve their HCV infection showed significantly lower anti-HBs titres than did responders with spontaneous $\mathrm{HCV}$ resolution. The latter group showed even higher anti-HBs titres than noninfected responders. Although HCV infection status divided HD patients into those with spontaneous $\mathrm{HCV}$ resolution and those showing persistent $\mathrm{HCV}$ infection, a significant positive correlation between IFN- $\lambda 3$ and anti$\mathrm{HBs}$ being generated in response to $\mathrm{HBV}$ vaccination was observed in both subgroups, independently of HCV outcome.

In the previous studies, homozygosity for the major allele (i.e., the CC genotype) in IFNL3 rs12979860 was attributed to spontaneous HCV clearance $[17,18,40]$ and resolution of HCV infection following treatment with pegylated IFN$\alpha$ and ribavirin [18, 19]. A study by Yu et al. [44] showed that IFNL3 rs8099917 affects spontaneous HCV clearance among Taiwanese HD patients. HD subjects bearing the rs8099917 allele $\mathrm{G}$ or the rs12979860 allele $\mathrm{T}$ had a lower chance of spontaneous HCV clearance [45]. In HD patients, comparison of responders and nonresponders to $\mathrm{HBV}$ vaccination revealed no significant differences in the IFNL3 genotype distribution; in $\mathrm{HBV}$-infected patients, differences in the distribution of IFNL3 variants were also not significant between anti-HBs-negative and anti-HBs-positive patients [45]. In this study, IFNL3 SNPs were significantly associated with neither HD patient outcomes nor circulating IFN$\lambda 3$, but homozygosity for the major alleles of rs8099917 or rs12979860 was associated with HCV clearance, as shown in many previous studies $[17,18,40,44,45]$.

In noninfected human hepatocytes, higher expression levels of IFNL3 were found in cells with the TT genotype of IFNL3 rs12979860 than in cells with the CC genotype [46]. Pretreatment liver biopsy specimens from chronic HCV patients revealed higher expression levels of IFN$\lambda 1$ and IFN- $\lambda 3$ in patients with the IFNL3 rs12979860 TT genotype than in those with the CC genotype. This higher expression of IFN- $\lambda 1$ and IFN- $\lambda 3$ was accompanied by higher expression of IFN- $\lambda$ receptor and antiviral IFN-stimulated genes [46]. These results seem to indicate that the TT genotype might be associated also with higher circulating IFN- $\lambda 3$, as suggested in low-flux HD patients in the current study. However, a combined population of subjects with persistent $\mathrm{HCV}$, individuals who spontaneously cleared $\mathrm{HCV}$, and healthy controls exhibited higher IFN- $\lambda 3$ serum levels in subjects showing major homozygosity of rs12979860 [18]. Interestingly, subjects with persistent HCV (an unfavourable outcome by our criteria) composed $63.0 \%$ of all subjects in this group.

In this study, we did not show association of IFNL3 rs8099917 with circulating IFN- $\lambda 3$ in the entire group of HD patients, which is in agreement with the results obtained by Aoki et al. [41] from Japanese patients with chronic hepatitis C. Lower intrahepatic expression of IFNL3 mRNA in $\mathrm{HCV}$-infected liver recipients and donors was demonstrated in subjects harbouring a variant allele of rs8099917 [47]. 
Expression levels of IFNL3 in peripheral blood mononuclear cells of HCV-infected subjects were also lower in patients who carried the variant alleles of $\operatorname{rs} 8099917[47,48]$ and were comparable with those in the liver [47]. In HD patients treated using low-flux dialysers, lower circulating IFN- $\lambda 3$ was also shown in bearers of a variant allele of rs8099917 [16]. On the other hand, subjects living in the Riyadh area of Saudi Arabia showed higher serum IFN- $\lambda 3$ levels if they were homozygous for a variant allele of rs8099917, whereas the major allele of rs8099917 was found to be correlated with reduced IFN- $\lambda 3$ serum levels [49].

We would like to stress that higher circulating IFN$\lambda 3$ levels were independently associated with favourable outcomes for HD patients with respect to responsiveness to HBV vaccination, and therefore with better protection of $\mathrm{HD}$ patients against $\mathrm{HBV}$, as well as with respect to selflimited HBV infection and spontaneous HCV clearance, and therefore with avoidance of serious liver complications related to persistent $\mathrm{HBV} / \mathrm{HCV}$ viremia (hepatic cirrhosis and hepatocellular carcinoma) [50-53]. The association of circulating IFN- $\lambda 3$ with the response status and the strength of the response to $\mathrm{HBV}$ vaccination or infection provides arguments for further studies dealing with the subject of why $\mathrm{HBV} / \mathrm{HBs} \mathrm{Ag}$ may be a trigger for IFN- $\lambda 3$ and anti-HBs development in some patients, whereas other subjects are not responsive.

\section{Study Limitations}

The main limitation of our study is the small number of patients in each subgroup. Therefore, only very evident differences surfaced as statistically significant. To show a statistically significant difference in IFN- $\lambda 3$ levels between HCV RNA-negative and HCV RNA-positive responders to $\mathrm{HBV}$ vaccination with a test power of $80 \%$, we would have needed a 2.1-fold greater number of responders in each group, assuming the same proportion of HCV RNA-positive and HCV RNA-negative responders as in the already-studied $\mathrm{HCV}$-exposed responders. Given the fine division of the patients into many subgroups, the number of patients in the entire sample needs to be much higher than what was available in the current study. Our study is regional; wholecountry assessment might be helpful in enrolling more satisfactory subgroups.

Determination of plasma IFN- $\lambda 3$ concentration is not routinely used and at present there are no recommendations of which test should be used for IFN- $\lambda 3$ level quantifications. In our studies ([16], this study), we applied the Chinese ELISA kit. The USA ELISA test for IFN- $\lambda 3$ was used by Li et al. [15] and Shi et al. [18]. Aoki et al. [41] quantified IFN- $\lambda 3$ by chemiluminescence enzyme immunoassay (CLEIA), which in healthy subjects yielded the lowest IFN- $\lambda 3$ levels compared with those presented by the other mentioned authors ([15, $16,18]$, this study). The Chinese ELISA kit provided lower IFN- $\lambda 3$ levels than those obtained by the USA ELISA kit. However, studies using the USA ELISA kit also did not show comparable results in healthy Chinese controls $[15,18]$. Therefore, IFN- $\lambda 3$ levels seem to depend on the method and even on the test used for IFN- $\lambda 3$ determination. We did not perform studies to establish the sensitivity and specificity of the used assay; however, further laboratory studies could specify which IFN- $\lambda 3$ concentrations describe true IFN- $\lambda 3$ levels.

\section{Conclusions}

(1) Circulating IFN- $\lambda 3$ is upregulated in HD responders to $\mathrm{HBV}$ vaccination compared with healthy responders.

(2) In $\mathrm{HD}$ patients, responders to $\mathrm{HBV}$ vaccination show higher circulating IFN- $\lambda 3$ levels than nonresponders.

(3) HD patients with self-limited HBV infection show higher IFN- $\lambda 3$ plasma concentrations than persistently HBsAg-positive subjects.

(4) In HD patients, spontaneous HCV clearance is associated with higher IFN- $\lambda 3$ levels; however, stimulation of the immune system by HBV vaccination for maintenance of protective anti-HBs titres may contribute to increases in IFN- $\lambda 3$ as anti-HBs production is associated with higher circulating IFN- $\lambda 3$.

(5) In HD patients, IFN- $\lambda 3$ is crucial for protection against $\mathrm{HBV}$ and for self-limitation of hepatotropic infections.

\section{Disclosure}

Results of this study will be presented at the American Society of Nephrology Kidney Week 2017 Annual Meeting, October 31-November 5, in New Orleans, LA.

\section{Conflicts of Interest}

The authors declare that they have no conflicts of interest.

\section{Acknowledgments}

The authors express their gratitude to the physicians of the dialysis centres for their consent in collecting the participants' data. This study was funded by the Poznan University of Medical Sciences, Poznan, Poland, Grant nos. 502-01-0222536303679 and 502-01-01124182-07474.

\section{References}

[1] A. Schweitzer, J. Horn, R. T. Mikolajczyk, G. Krause, and J. J. Ott, "Estimations of worldwide prevalence of chronic hepatitis $B$ virus infection: a systematic review of data published between 1965 and 2013," The Lancet, vol. 386, no. 10003, pp. 1546-1555, 2015.

[2] K. M. Hanafiah, J. Groeger, A. D. Flaxman, and S. T. Wiersma, "Global epidemiology of hepatitis C virus infection: new estimates of age-specific antibody to HCV seroprevalence," Hepatology, vol. 57, no. 4, pp. 1333-1342, 2013.

[3] B. Rutkowski, G. Korejwo, S. Czekalski, and S. Bautembach, "Hemodialysis," in Report on Renal Replacement Therapy in Poland-2010, B. Rutkowski, M. Lichodziejewska-Niemierko, R. Grenda, S. Czekalski, M. Durlik, and S. Bautembach, Eds., pp. 7-33, Gdańsk, 2013. 
[4] M. Rosińska, N. Parda, and M. Stępień, "Hepatitis C in Poland in 2014," Przeglad Epidemiologiczny, vol. 70, pp. 386-394, 2016.

[5] A. E. Grzegorzewska, "Hepatitis B vaccination in chronic kidney disease patients: a call for novel vaccines," Expert Review of Vaccines, vol. 13, no. 11, pp. 1317-1326, 2014.

[6] A. E. Grzegorzewska, "Prophylactic vaccinations in chronic kidney disease: current status," Human Vaccines \& Immunotherapeutics, vol. 11, no. 11, pp. 2599-2605, 2015.

[7] J. D. Harnett, P. S. Parfrey, M. Kennedy, J. B. Zeldis, T. I. Steinman, and R. D. Guttmann, "The long-term outcome of hepatitis B infection in hemodialysis patients," American Journal of Kidney Diseases, vol. 11, no. 3, pp. 210-213, 1988.

[8] K. Okuda, H. Hayashi, K. Yokozeki, S. Kobayashi, T. Kashima, and Y. Irie, "Acute hepatitis $\mathrm{C}$ among renal failure patients on chronic haemodialysis," Journal of Gastroenterology and Hepatology, vol. 13, no. 1, pp. 62-67, 1998.

[9] S. V. Kotenko, G. Gallagher, V. V. Baurin et al., "IFN- $\lambda$ s mediate antiviral protection through a distinct class II cytokine receptor complex," Nature Immunology, vol. 4, no. 1, pp. 69-77, 2003.

[10] P. Sheppard, W. Kindsvogel, W. Xu et al., "IL-28, IL-29 and their class II cytokine receptor IL-28R," Nature Immunology, vol. 4, no. 1, pp. 63-68, 2003.

[11] T. Marcello, A. Grakoui, G. Barba-Spaeth et al., "Interferons alpha and lambda inhibit hepatitis $C$ virus replication with distinct signal transduction and gene regulation kinetics," Gastroenterology, vol. 131, no. 6, pp. 1887-1898, 2006.

[12] M. D. Robek, B. S. Boyd, and F. V. Chisari, "Lambda interferon inhibits hepatitis B and C virus replication," Journal of Virology, vol. 79, no. 6, pp. 3851-3854, 2005.

[13] S. E. Doyle, H. Schreckhise, K. Khuu-Duong et al., "Interleukin29 uses a type 1 interferon-like program to promote antiviral responses in human hepatocytes," Hepatology, vol. 44, no. 4, pp. 896-906, 2006.

[14] C. Kelly, P. Klenerman, and E. Barnes, "Interferon lambdas: the next cytokine storm," Gut, vol. 60, no. 9, pp. 1284-1293, 2011.

[15] W. Li, Y. Jiang, Q. Jin et al., "Expression and gene polymorphisms of interleukin 28B and hepatitis B virus infection in a Chinese Han population," Liver International, vol. 31, no. 8, pp. 1118-1126, 2011.

[16] A. E. Grzegorzewska, M. K. Świderska, A. Mostowska, W. Warchol, and P. P. Jagodziński, "Antibodies to HBV surface antigen in relation to interferon- $\lambda 3$ in hemodialysis patients," Vaccine, vol. 34, no. 41, pp. 4866-4874, 2016.

[17] D. L. Thomas, C. L. Thio, M. P. Martin et al., "Genetic variation in IL28B and spontaneous clearance of hepatitis C virus," Nature, vol. 461, no. 7265, pp. 798-801, 2009.

[18] X. Shi, Y. Pan, M. Wang et al., "IL28B genetic variation is associated with spontaneous clearance of hepatitis $\mathrm{C}$ virus, treatment response, serum IL-28B levels in Chinese population," PLoS ONE, vol. 7, no. 5, Article ID e37054, 2012.

[19] D. Ge, J. Fellay, A. J. Thompson et al., "Genetic variation in IL28B predicts hepatitis C treatment-induced viral clearance," Nature, vol. 461, no. 7262, pp. 399-401, 2009.

[20] S. Tang, M. Yue, J. Wang et al., "Associations of IFN- $\gamma$ rs2430561 T/A, IL28B rs12979860 C/T and ER $\alpha$ rs2077647 T/C polymorphisms with outcomes of hepatitis B virus infection: a metaanalysis," Journal of Biomedical Research, vol. 28, no. 6, pp. 484493, 2014.

[21] E. E. Mast, H. S. Margolis, A. E. Fiore et al., "Advisory Committee on Immunization Practices (ACIP). Advisory Committee on Immunization Practices (ACIP) Centers for Disease Control and Prevention (CDC). A comprehensive immunization strategy to eliminate transmission of hepatitis B virus infection in the United States: recommendations of the Advisory Committee on Immunization Practices (ACIP) Part II: immunization of adults," MMWR Recommendations and Reports, vol. 55, no. 16, pp. 1-33, 2006.

[22] R Core Team, A Language and Environment for Statistical Computing, R Foundation for Statistical Computing, Vienna, Austria, 2017, https://www.R-project.org/.

[23] J. L. Rukov, E. Gravesen, M. L. Mace et al., "Effect of chronic uremia on the transcriptional profile of the calcified aorta analyzed by RNA sequencing," American Journal of PhysiologyRenal Physiology, vol. 310, no. 6, pp. F477-F491, 2016.

[24] N. D. Vaziri, M. V. Pahl, A. Crum, and K. Norris, "Effect of uremia on structure and function of the immune system," Journal of Renal Nutrition, vol. 22, no. 1, pp. 149-156, 2012.

[25] G. Pertosa, G. Grandaliano, L. Gesualdo, and F. P. Schena, "Clinical relevance of cytokine production in hemodialysis," Kidney International Supplements, vol. 58, no. 76, pp. S104-S111, 2000.

[26] D. Teta, "Adipokines as uremic toxins," Journal of Renal Nutrition, vol. 22, no. 1, pp. 81-85, 2012.

[27] H. Mabuchi and H. Nakahashi, "Analysis of small peptides in uremic serum by high-performance liquid chromatography," Journal of Chromatography B: Biomedical Sciences and Applications, vol. 228, no. C, pp. 292-297, 1982.

[28] J. M. Fox, J. M. Crabtree, L. K. Sage, S. M. Tompkins, and R. A. Tripp, "Interferon lambda upregulates IDO1 expression in respiratory epithelial cells after influenza virus infection," Journal of Interferon \& Cytokine Research, vol. 35, no. 7, pp. 554$562,2015$.

[29] T. Eleftheriadis, T. Sparopoulou, G. Antoniadi, V. Liakopoulos, I. Stefanidis, and G. Galaktidou, "Suppression of humoral immune response to hepatitis B surface antigen vaccine in BALB/C mice by 1-methyl-tryptophan co-administration," DARU Journal of Pharmaceutical Sciences, vol. 19, no. 3, pp. 236239, 2011.

[30] H. Xu, T. B. Oriss, M. Fei et al., "Indoleamine 2,3-dioxygenase in lung dendritic cells promotes Th2 responses and allergic inflammation," Proceedings of the National Acadamy of Sciences of the United States of America, vol. 105, no. 18, pp. 6690-6695, 2008.

[31] H. Xu, G. X. Zhang, B. Ciric, and A. Rostami, "IDO: a doubleedged sword for TH1/TH2 regulation," Immunology Letters, vol. 121, no. 1, pp. 1-6, 2008.

[32] M. P. Morrow, P. Pankhong, D. J. Laddy et al., "Comparative ability of IL-12 and IL-28B to regulate Treg populations and enhance adaptive cellular immunity," Blood, vol. 113, no. 23, pp. 5868-5877, 2009.

[33] G. Lonnemann, D. Novick, M. Rubinstein, and C. A. Dinarello, "Interleukin-18, interleukin-18 binding protein and impaired production of interferon-g in chronic renal failure," Clinical Nephrology, vol. 60, no. 5, pp. 327-334, 2003.

[34] M. P. Morrow, J. Yan, P. Pankhong et al., "IL-28B/IFN $\lambda$-3 drives granzyme B loading and significantly increases CTL killing activity in macaques," Molecular Therapy, vol. 18, no. 9, pp. 17141723, 2010.

[35] S. Sato, K. Li, T. Kameyama et al., "The RNA Sensor RIGI Dually Functions as an Innate Sensor and Direct Antiviral Factor for Hepatitis B Virus," Immunity, vol. 42, no. 1, pp. 123132, 2015. 
[36] S. Yoshio, M. Sugiyama, H. Shoji et al., "Indoleamine-2,3dioxygenase as an effector and an indicator of protective immune responses in patients with acute hepatitis B," Hepatology, vol. 63, no. 1, pp. 83-94, 2016.

[37] P. Lampertico, M. Viganò, C. Cheroni et al., "IL28B polymorphisms predict interferon-related hepatitis B surface antigen seroclearance in genotype $\mathrm{D}$ hepatitis $\mathrm{B}$ e antigen-negative patients with chronic hepatitis B," Hepatology, vol. 57, no. 3, pp. 890-896, 2013.

[38] Z.-F. Meng, H.-J. Wang, X. Yao et al., "Immunization with HBsAg-Fc fusion protein induces a predominant production of Thl cytokines and reduces HBsAg level in transgenic mice," Chinese Medical Journal, vol. 125, no. 18, pp. 3266-3272, 2012.

[39] S. Wieland, Z. Makowska, B. Campana et al., "Simultaneous detection of hepatitis $C$ virus and interferon stimulated gene expression in infected human liver," Hepatology, vol. 59, no. 6, pp. 2121-2130, 2014.

[40] P.-Y. Bochud, S. Bibert, Z. Kutalik et al., "IL28B alleles associated with poor hepatitis $\mathrm{C}$ virus $(\mathrm{HCV})$ clearance protect against inflammation and fibrosis in patients infected with non-1 HCV genotypes," Hepatology, vol. 55, no. 2, pp. 384-394, 2012.

[41] Y. Aoki, M. Sugiyama, K. Murata et al., "Association of serum IFN- $\lambda 3$ with inflammatory and fibrosis markers in patients with chronic hepatitis C virus infection," Journal of Gastroenterology, vol. 50, no. 8, pp. 894-902, 2015.

[42] A. E. Grzegorzewska, M. K. Świderska, and W. Warchoł, "Antibodies to hepatitis B virus surface antigen and survival of hemodialysis patients-a prospective study," Expert Review of Vaccines, vol. 15, no. 8, pp. 1063-1074, 2016.

[43] J. P. Moorman, C. L. Zhang, L. Ni et al., "Impaired hepatitis $B$ vaccine responses during chronic hepatitis $C$ infection: Involvement of the PD-1 pathway in regulating CD4+ T cell responses," Vaccine, vol. 29, no. 17, pp. 3169-3176, 2011.

[44] M.-L. Yu, C.-Y. Dai, C.-F. Huang et al., "High hepatitis B virus surface antigen levels and favorable interleukin 28B genotype predict spontaneous hepatitis C virus clearance in uremic patients," Journal of Hepatology, vol. 60, no. 2, pp. 253-259, 2014.

[45] A. E. Grzegorzewska, E. Jodłowska, A. Mostowska, and P. P. Jagodziński, "Effect of interferon $\lambda 3$ gene polymorphisms, rs8099917 and rs12979860, on response to hepatitis B virus vaccination and hepatitis $\mathrm{B}$ or $\mathrm{C}$ virus infections among hemodialysis patients," Polskie Archiwum Medycyny Wewnętrznej, vol. 125, no. 12, pp. 894-902, 2015.

[46] P. Ferraris, P. K. Chandra, R. Panigrahi et al., "Cellular mechanism for impaired hepatitis c virus clearance by interferon associated with IFNL3 gene polymorphisms relates to intrahepatic interferon- $\lambda$ expression," The American Journal of Pathology, vol. 186, no. 4, pp. 938-951, 2016.

[47] T. Fukuhara, A. Taketomi, T. Motomura et al., "Variants in IL28B in liver recipients and donors correlate with response to peg-interferon and ribavirin therapy for recurrent hepatitis C," Gastroenterology, vol. 139, no. 5, pp. 1577.e3-1585.e3, 2010.

[48] Y. Tanaka, N. Nishida, M. Sugiyama et al., "Genome-wide association of IL28B with response to pegylated interferon- $\alpha$ and ribavirin therapy for chronic hepatitis C," Nature Genetics, vol. 41, no. 10, pp. 1105-1109, 2009.

[49] A. Al-Qahtani, M. Al-Anazi, and A. A. Abdo, "Correlation between genetic variations and serum level of interleukin $28 \mathrm{~B}$ with virus genotypes and disease progression in chronic hepatitis C virus infection," Journal of Immunology Research, vol. 2015, Article ID 768470, 10 pages, 2015.
[50] T. Poynard, P. Bedossa, and P. Opolon, "Natural history of liver fibrosis progression in patients with chronic hepatitis C," The Lancet, vol. 349, no. 9055, pp. 825-832, 1997.

[51] P. Nahon, A. Sutton, P. Rufat et al., "Chemokine system polymorphisms, survival and hepatocellular carcinoma occurrence in patients with hepatitis C virus-related cirrhosis," World Journal of Gastroenterology, vol. 14, no. 5, pp. 713-719, 2008.

[52] J. Fung, C.-L. Lai, D. But, D. Wong, T.-K. Cheung, and M.-F. Yuen, "Prevalence of fibrosis and cirrhosis in chronic hepatitis B: implications for treatment and management," American Journal of Gastroenterology, vol. 103, no. 6, pp. 1421-1426, 2008.

[53] H. B. El-Serag, "Epidemiology of viral hepatitis and hepatocellular carcinoma," Gastroenterology, vol. 142, no. 6, pp. 12641273, 2012. 


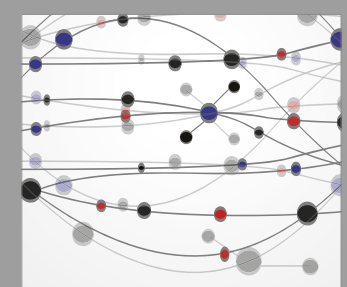

The Scientific World Journal
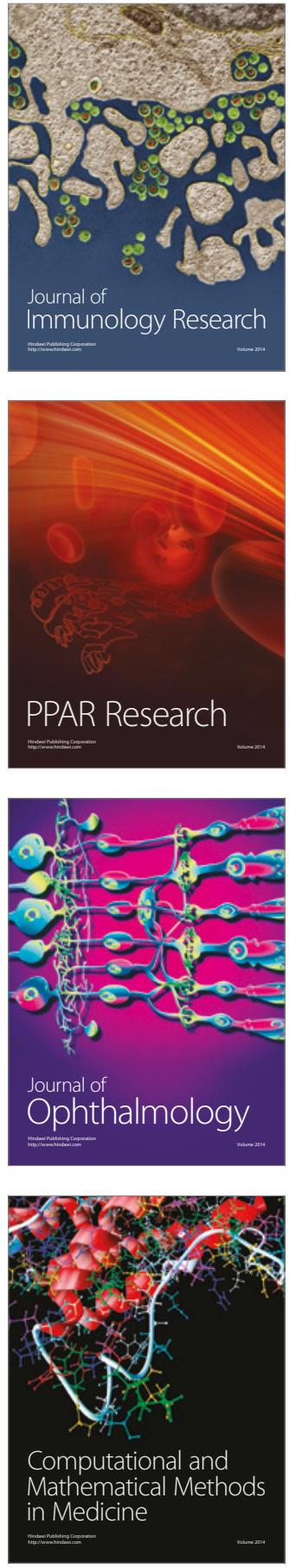

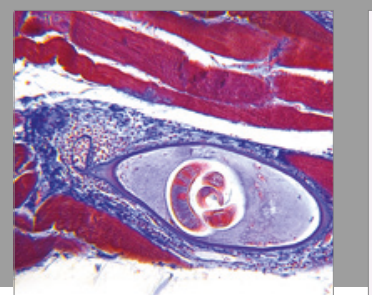

Gastroenterology Research and Practice
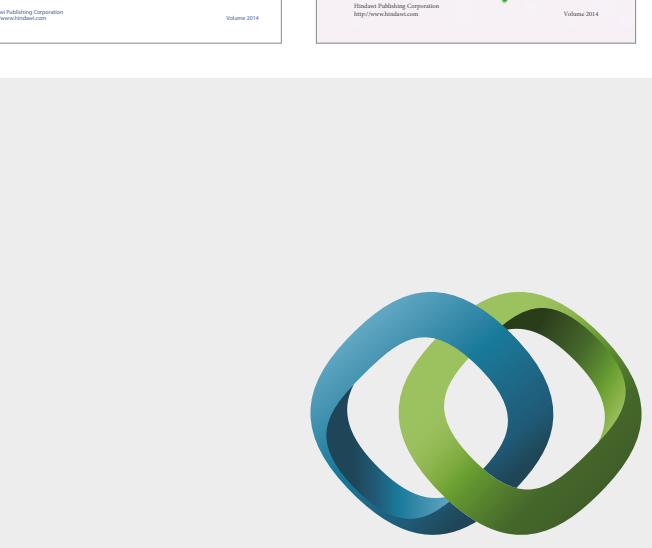

\section{Hindawi}

Submit your manuscripts at

https://www.hindawi.com
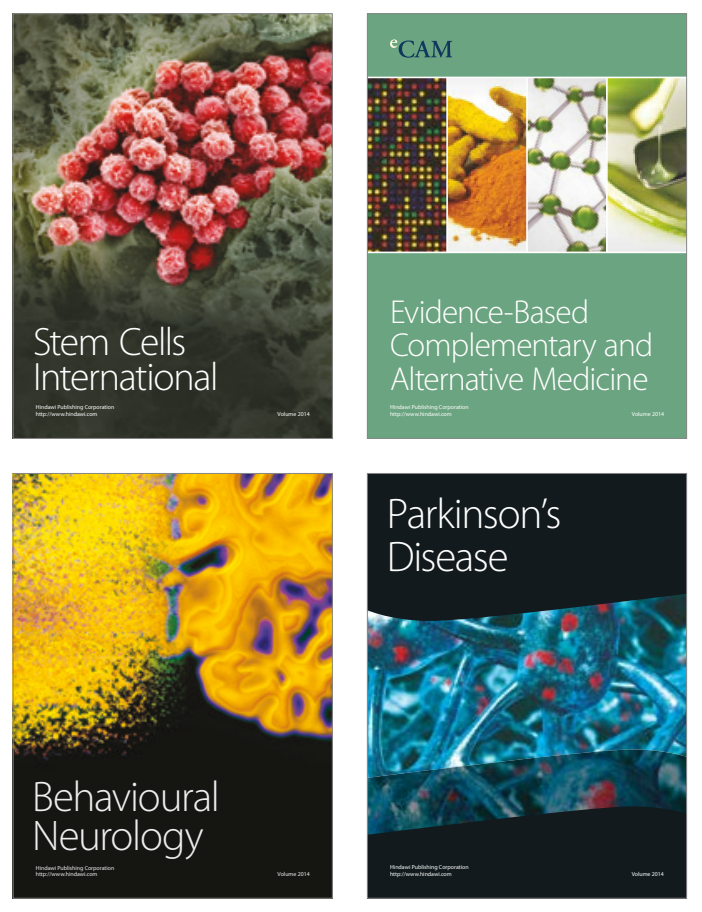
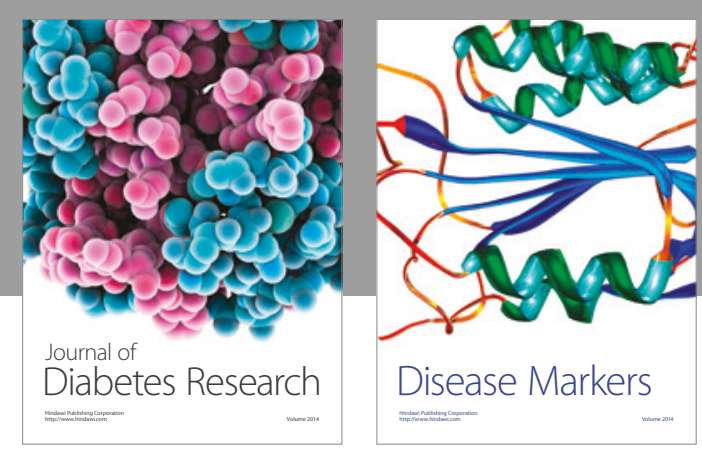

Disease Markers
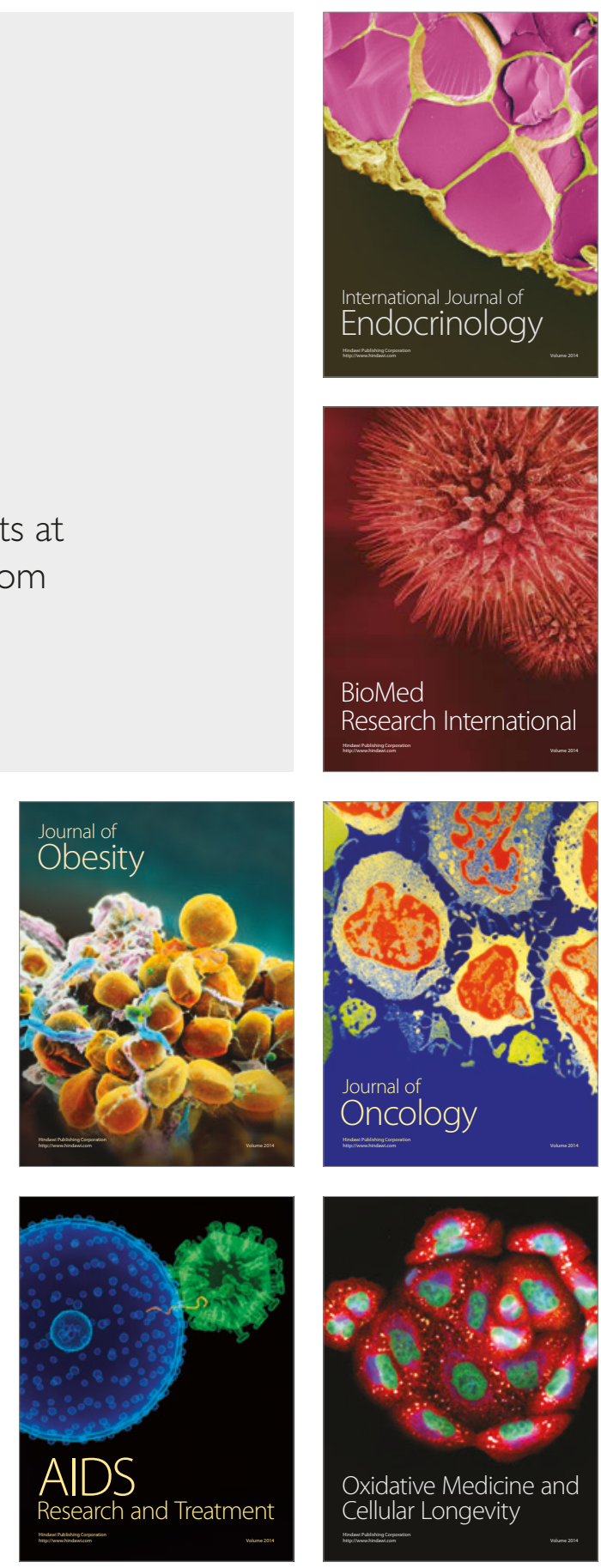